\title{
Impact of the Film Array Meningitis/ Encephalitis panel in adults with meningitis and encephalitis in Colombia
}

\section{Original Paper}

Cite this article: Díaz KMO, Piedrahíta JAA, Suárez Brochero OF, Granada DO, Barón LM, Bonilla IC, Hasbun R (2020). Impact of the Film Array Meningitis/Encephalitis panel in adults with meningitis and encephalitis in Colombia. Epidemiology and Infection 148, e173, 1-6. https://doi.org/10.1017/S0950268820001648

Received: 3 January 2020

Revised: 12 July 2020

Accepted: 19 July 2020

\section{Key words:}

Antibiotic utilisation; Colombia; encephalitis; meningitis; meningitis-encephalitis multiplex PCR

Author for correspondence:

Rodrigo Hasbun,

E-mail: Rodrigo.Hasbun@uth.tmc.edu

\section{Karen Melissa Ordóñez Díaz ${ }^{1}$, John Alexander Alzate Piedrahíta ${ }^{1}$, Oscar Felipe Suárez Brocheroํㅡ, Daniel Orozco Granada ${ }^{1}$, Laura Marcela Barón ${ }^{1}$, Isabella Cortés Bonilla ${ }^{2}$ and Rodrigo Hasbun ${ }^{3}$ (b)}

\footnotetext{
${ }^{1}$ Hospital Universitario San Jorge, Pereira, Colombia; ${ }^{2}$ Universidad Tecnológica de Pereira, Pereira, Colombia and ${ }^{3}$ UT Health McGovern Medical School, Houston, TX, USA
}

\begin{abstract}
The Biofire ${ }^{\varpi}$ Film Array Meningitis Encephalitis (FAME) panel can rapidly diagnose common aetiologies but its impact in Colombia is unknown. A retrospective study of adults with CNS infections in one tertiary hospital in Colombia. The cohort was divided into two time periods: before and after the implementation of the Biofire FAME panel in May 2016. A total of 98 patients were enrolled, 52 and 46 were enrolled in the Standard of Care (SOC) group and in the FAME group, respectively. The most common comorbidity was human immunodeficiency virus infection (47.4\%). The median time to a change in therapy was significantly shorter in the FAME group than in the SOC group $(3 v s .137 .3 \mathrm{~h}, P<0.001)$. This difference was driven by the timing to appropriate therapy $(2.1 v s .195 \mathrm{~h}, P<0.001)$ by identifying viral aetiologies. Overall outcomes and length of stay were no different between both groups $(P>0.2)$. The FAME panel detected six aetiologies that had negative cultures but missed identifying one patient with Cryptococcus neoformans. The introduction of the Biofire FAME panel in Colombia has facilitated the identification of viral pathogens and has significantly reduced the time to the adjustment of empirical antimicrobial therapy.
\end{abstract}

(C) The Author(s), 2020. Published by Cambridge University Press. This is an Open Access article, distributed under the terms of the Creative Commons Attribution licence (http://creativecommons.org/licenses/by/4.0/), which permits unrestricted re-use, distribution, and reproduction in any medium, provided the original work is properly cited.

\section{CAMBRIDGE} UNIVERSITY PRESS

\section{Introduction}

Meningitis and encephalitis continue to be associated with significant neurological morbidity and mortality [1], so establishing a cause and administering prompt antimicrobial therapy is crucial in improving clinical outcomes in several aetiologies [2, 3]. Unfortunately, rapidly establishing an aetiological diagnosis in public hospitals in Colombia is not easy, given that most of these facilities do not have inhouse diagnostic tools such as specific polymerase chain reaction (PCR) for viruses and bacteria or latex for the detection of the capsular antigen of Cryptococcus spp. The performance of molecular diagnostic tests requires the shipping of the sample to a reference laboratory in either Medellin or Bogota with an approximate delay of 10-14 days to receive the results. Therefore, the possibilities of determining the causal agent were subject to the yield of traditional cultures, interpretation of the cerebrospinal fluid (CSF) cytochemistry and clinical findings prompting empirical therapy for the majority of patients [4]. In patients with bacterial meningitis, the sensitivity of the CSF Gram stain ranges from $10 \%$ to $93 \%$ and the CSF cultures between $50 \%$ and $85 \%$ depending on the pathogen, country of the study and by the receipt of previous antibiotic therapy [5]. This diagnostic uncertainty and the fear of an adverse outcome lead to an indiscriminate use of antimicrobials in the majority of patients. Clinicians prefer to initiate and maintain broad-spectrum therapies that include antibiotics, antivirals and in the case of HIV-positive antifungal patients, which increases not only treatment costs but also the risk of adverse events such as nephrotoxicity and Clostridium difficile diarrhoea [6].

The Biofire ${ }^{\circledast}$ Film Array Meningitis Encephalitis (FAME) panel is a multiplex PCR tool that utilises a sample of $200 \mu \mathrm{l}$ of CSF to identify in $1 \mathrm{~h}$ the presence of 14 pathogens (Escherichia coli K1, Haemophilus influenzae, Listeria monocytogenes, Neisseria meningitidis, Streptococcus agalactiae, S. pneumoniae, cytomegalovirus (CMV), enterovirus (EV), herpes simplex virus 1 (HSV-1), herpes simplex virus 2 (HSV-2), human herpes virus 6 (HHSV-6), human parechovirus (HPeV), varicella zoster (VZV), Cryptococcus neoformans/C. gattii) that was approved by the Federal Drug Administration (FDA) since 2015. Diagnostic correlation studies with CSF sample banks positive for the targets identified by the test have an agreement of $>90 \%$ $[7,8]$. The main limitation of this panel is with C. neoformans where it can be as low as $50 \%$ [8]. So far, studies have been carried out mainly in the pediatric population and in immunocompetent patients with overall good performance, although only approximately 
$25 \%$ of the film array panels are positive $[9,10]$. Currently, there are no studies evaluating the performance of this panel in Latin America.

The Hospital San Jorge de Pereira is a tertiary care public hospital in the central-western region of Colombia, where the prevalence of HIV disease is one of the highest in the country. One of the most challenging infections is meningitis and encephalitis as the diagnosis is unknown in the majority of patients. In 2016, the Biofire ${ }^{\varpi}$ FAME panel was introduced. The aim of the present study was to evaluate the clinical impact of the introduction of this test in adults with meningitis and encephalitis.

\section{Materials and methods}

The study was divided into two cohorts of adult patients with meningitis or encephalitis: before (Standard of Care (SOC) group) and after the introduction of the Biofire ${ }^{\infty}$ FAME panel (FAME group). The inclusion criteria include adults (age $>17$ years) with a diagnostic suspicion of neuroinfection who had a CSF analysis and had complete medical records. Only the first episode of infection was taken into account. Data were collected through an electronic format which include demographic variables (age, sex), clinical (comorbidities), paraclinical (CSF cytochemical), culture results, FAME panel test result, days of antimicrobial treatment, difference in hours to make adjustments to the antimicrobial with microbiological test results (timeframe between the lumbar puncture to escalation, de-escalation or discontinuation of antimicrobial therapy), days were evaluated of total hospitalisation, days of hospitalisation in ICU, state of discharge (alive or dead). The cost of the diagnostic studies was calculated for both groups according to the protocol for the study of patients with clinical suspicion of central nervous system infection. The SOC for meningitis diagnosis included blood cultures, computed tomography scans with magnetic resonance imaging testing when encephalitis was suspected. The study of CSF included culture, Gram stain, India ink stain, fungal culture. If the patient presented HIV diagnosis, the study of CSF included CMV PCR and cryptococcal antigen test.

FAME studies included blood cultures, computed tomography scans, magnetic resonance imaging testing when encephalitis was suspected. The study of CSF included culture, Gram stain, India ink stain, Biofire FAME panel. HIV and non-HIV patients were studied in the same way.

According to the protocol at the hospital, patients with clinical suspicion of neuroinfection were assessed by neurology and infectious diseases. Antimicrobial treatment was initiated according to guidelines (ceftriaxone and vancomycin for bacterial meningitis, amphotericin B-antituberculous-ceftriaxone-ampicillin for patients with HIV disease, and acyclovir for suspected encephalitis) and a CSF study was performed to perform diagnostic tests. The FAME panel was requested by either the neurologist or the infectious diseases specialist, the latter one interpreted the results and adjusted the medical management as soon as the panel was done. An appropriate antimicrobial therapy was defined as a therapy initiated with a diagnosis of meningitis/encephalitis constructed by clinical signs and symptoms, laboratory findings compatible with infection, with adjustment according to Gram stain results, culture results and PCR results when those were available. The antimicrobial stewardship programme headed by an infectious diseases specialist determined the concept of appropriate therapy.
Table 1. Comparison of baseline characteristics and outcomes between Standard of Care (SOC) and Film Array Meningitis-Encephalitis (FAME) groups

\begin{tabular}{|c|c|c|c|}
\hline Baseline variables & $\begin{array}{c}\text { SOC } \\
(n=52)\end{array}$ & $\begin{array}{l}\text { FAME } \\
(n=46)\end{array}$ & $P$ value \\
\hline $\begin{array}{l}\text { Median age } \\
\text { (years, range) }\end{array}$ & $36(25.5-45.5)$ & $43(34-55)$ & $0.040 \dagger$ \\
\hline Female sex, $n(\%)$ & $8(15.4)$ & $14(30.4)$ & 0.075 \\
\hline \multicolumn{4}{|l|}{ Clinical characteristics, n (\%) } \\
\hline Headache & $40(76.9)$ & $29(63)$ & 0.133 \\
\hline Altered mental status & $28(53.8)$ & $29(63)$ & 0.357 \\
\hline Meningeal signs & $27(51.9)$ & $23(50)$ & 0.849 \\
\hline Seizures & $22(42.3)$ & $17(36.9)$ & 0.589 \\
\hline Focal neurological exam & $6(11.5)$ & $7(15.2)$ & 0.592 \\
\hline $\begin{array}{l}\text { Charlson comorbidity } \\
\text { score (median, IQR) }\end{array}$ & 6 (IQR 1- 7) & 6 (IQR 1-7) & $0.956 \dagger$ \\
\hline $\begin{array}{l}\text { Human immunodeficiency } \\
\text { virus (HIV positive), } n(\%)\end{array}$ & $25(48)$ & $23(50)$ & 0.849 \\
\hline \multicolumn{4}{|l|}{ Comorbidities, $n(\%)$} \\
\hline Diabetes mellitus & $3(5.7)$ & $4(8.7)$ & 0.575 \\
\hline $\begin{array}{l}\text { Chronic renal failure } \\
\text { (stage IV-V) }\end{array}$ & $1(1.9)$ & $3(6.5)$ & 0.251 \\
\hline Tuberculosis & $5(9.6)$ & $5(10.8)$ & 0.930 \\
\hline Solid tumours & $1(1.9)$ & $4(8.7)$ & 0.128 \\
\hline Neutropenia & $1(1.9)$ & 0 & - \\
\hline
\end{tabular}

$\mathrm{IQR}$, interquartile range.

The study was approved by the Institutional Review Board of the Hospital San Jorge de Pereira.

\section{Statistical analysis}

The results were described as frequencies and medians with interquartile ranges between FAME and SOC groups. Statistical differences were assessed by $\chi^{2}$ and Fisher's exact test, when comparing categorical variables, and by Mann-Whitney $U$ test for comparing medians. Main outcomes were time to appropriate therapy, length of stay and in-hospital mortality. Glasgow Outcome Scale (GOS), use of diagnostic methods (MRI, head CT scan, blood culture bottles) and use of antimicrobials were secondary outcomes.

\section{Results}

Of 118 patients reviewed, 20 patients were excluded because a neuroinfection was ruled out. A total of 98 patients met the inclusion criteria for the study. Of these, 52 (52.5\%) patients were in the SOC group before the implementation of the panel and 46 $(47.5 \%)$ patients were in the FAME group. The demographic characteristics and outcomes between the two groups of patients are shown in Table 1. There were no differences in regards to sex, clinical characteristics, comorbidities and outcomes $(P>$ $0.05)$ but patients in the FAME group were older $(P=0.04)$.

As shown in Table 2, there were no differences in the CSF profile between the SOC and FAME groups $(P>0.05)$. A positive CSF India ink and Gram stain were seen in $5 \%$ and $12 \%$ of patients, respectively, with no differences between the two groups $(P>$ 
Table 2. Cerebrospinal fluid (CSF) results and aetiologies between Standard of Care (SOC) and Film Array Meningitis-Encephalitis (FAME) groups

\begin{tabular}{lccc}
\hline Variables & SOC $(n=52)$ & FAME $(n=46)$ & $P$ value \\
\hline Median CSF WBC (cell/ml) & $12(1-69)$ & $21.5(0-152)$ & 0.441 \\
\hline Median neutrophil per cent (\%, IQR) & $0(0-25)$ & $10(0-30)$ & 0.201 \\
\hline Median CSF protein (g/dl, IQR) & $104(29-228)$ & $46-223)$ & 0.363 \\
\hline Median CSF glucose (m/dl, IQR) & $48(30-60)$ & $43(30-54)$ & 0.385 \\
\hline Positive Gram stain, $n(\%)$ & $8(15.4)$ & $4(8.7)$ & 0.313 \\
\hline Positive Indian Ink, $n(\%)$ & $4(7.7)$ & $1(2.1)$ & $3(6.5)$ \\
\hline Positive CSF culture, $n(\%)$ & $11(21.1)$ & $17(36.9)$ & 0.735 \\
\hline Aetiologies, $n$ (\%) & $14^{\mathrm{a}}(26.9)$ & $5^{\mathrm{c}}$ & \\
\hline Bacterial meningitis & $7^{\mathrm{b}}$ & 6 & 0.039 \\
\hline Cryptococcus neoformans & 7 & 3 & 0.286 \\
\hline Cytomegalovirus & 0 & $3^{\mathrm{d}}$ & \\
\hline Herpes simplex virus & 0 & 6 & \\
\hline
\end{tabular}

WBC, white blood cell; IQR, interquartile range; $\mathrm{mg}$, milligram; dl, decilitre; g, gram.

${ }^{a}$ In SOC group, the isolation of $E$. coli was in blood cultures, 1 diagnosis of $C$. neoformans was by latex antigen (negative cultures), 6 positive CSF cultures for $C$. neoformans, the other bacterial isolates were identified in CSF culture only.

${ }^{b}$ S. pneumoniae (2), K. pneumoniae (1), E. coli (1). Gram-positive bacilli (1), Gram-positive cocci (1), Gram-negative cocci (1).

'S. pneumoniae (2), N. meningitidis (1), H. influenzae (1), Gram-positive cocci (1).

${ }^{\mathrm{d}}$ Herpes simplex virus type 1 (1), Herpes simplex virus type 2 (2).

02). A positive CSF culture was seen more frequently in the SOC group (21\% vs 6.5\%, $P=0.039)$.

As shown in Table 2, 14 (27\%) of the SOC had an aetiology identified and 17 (36.9\%) in the FAME group. Only the FAME group identified viral aetiologies (three with cytomegalovirus and three with herpes simplex viruses). All other aetiologies in both groups were either bacterial or fungal. As shown in Table 3, only two out of four positive FAME panels for bacteria were also detected by cultures. In regards to $C$. neoformans: four positive FAME had negative CSF cultures, one patient had both a positive FAME and culture and one patient had a negative FAME but a positive CSF culture for C. neoformans.

Overall outcomes and length of stay in the hospital or in the intensive care unit were no different between both groups $(P>$ 0.2 ) (see Table 4). The median time to a change in therapy was significantly shorter in the FAME group than in the SOC group ( 3 vs. $137.3 \mathrm{~h}, P<0.001)$. This difference was driven by the timing to appropriate therapy $(2.1 v s .195 \mathrm{~h}, P<0.001)$ mostly driven by identifying viral aetiologies such as CMV and HSV in a timely fashion (Table 5). The prolonged delay initiation in the SOC group was seen mainly in non-HIV patients with cryptococcal meningitis, and in some patients with viral encephalitis not suspected by clinical presentation or CSF characteristics.

Furthermore, there was a trend that inadequate antimicrobial therapy was discontinued in a timelier manner in the FAME group (19.1 vs. $92 \mathrm{~h}, P=0.05$ ). Patients in the SOC group also had more blood cultures done than in those in the FAME group $(P=0.045)$. Patients in the FAME group had more magnetic resonance imaging of the brain, a finding associated with the acquisition of the resource by the institution during this period. As shown in Table 6, there were no differences in the use and duration of the different antimicrobial therapies between both groups $(P>0.5)$. The median cost of antimicrobial treatment in the SOC group was US $\$ 456.48$ (US $\$ 31.01-U S \$ 854.56$ ) per patient treatment course compared to the median cost of antimicrobial treatment in FAME group that was US\$309.81 (US\$0-US
\$694.74) per patient $(P=0.184)$. When including the cost of diagnostic testing, the median cost per patient using the SOC was US $\$ 755.4$ (US $\$ 251.58-$ US $\$ 1128.82$ ) per treatment course vs. US $\$ 602.09$ (US\$398.08-US\$1071.34) in the FAME group ( $P$ 0.685).

\section{Discussion}

The present study was conducted to evaluate the clinical impact of the implementation of the FAME panel as part of the protocol for the study and management of adult patients with meningitis or encephalitis in a public hospital in Colombia. The proportion of positive results of the FAME panel in this study was higher (34.7\%) than seen in other previous studies (8.7\% [7]; 10.4\% [11]; $12.7 \%$ [9]).

We had no false-positive FAME results in our study. All of the positive results had either a consistent clinical and CSF picture or an isolation in culture (for fungi and bacteria). No additional PCR tests were performed to confirm the viral aetiologies. Unlike other studies done in the USA where the main aetiologies were viruses, the main aetiological agent in our study before and after the establishment of the panel was C. neoformans. This finding is related to the high prevalence of HIV disease in the population studied $(\sim 50 \%)$.

Only patients in the FAME group were able to identify viral aetiologies such as CMV and HSV. Furthermore, the FAME panel was able to detect bacteria and C. neoformans in patients with negative CSF and blood cultures. This could be in some patients due to previous antibiotic therapy. We also observed a false-negative result in a patient with culture-confirmed cryptococcal meningitis. The presence of false negatives in cryptococcal meningitis in patients studied with the ME panel coincides with recent literature $[8,12]$, but differs from that found in the studies conducted in Uganda where a high concordance rate was seen with culture and latex antigen [13]. Given the severity of the outcomes that may result from stopping an antifungal therapy due to a false-negative FAME panel, it is recommended that if the 
Table 3. Concordance of the Biofire Film Array Meningitis Encephalitis (FAME) with blood and cerebrospinal fluid (CSF) cultures

\begin{tabular}{|c|c|c|}
\hline Aetiology & FAME result & Culture results \\
\hline Streptococcus pneumoniae & S. pneumoniae & S. pneumoniae on CSF only \\
\hline Streptococcus pneumoniae & S. pneumoniae & Negative on blood and CSF \\
\hline Neisseria meningitidis & N. meningitidis & N. meningitidis isolated only in blood culture \\
\hline Haemophilus influenzae & H. influenzae & Negative blood and CSF \\
\hline Gram-positive cocci (Gram stain only) & Negative & Negative blood and CSF \\
\hline Cryptococcus neoformans & Negative & C. neoformans isolated in CSF only \\
\hline Cryptococcus neoformans & C. neoformans & C. neoformans isolated in CSF only \\
\hline Cryptococcus neoformans (4) & C. neoformans & Negative blood and CSF \\
\hline
\end{tabular}

Table 4. Lengths of stay, mortality, time to appropriate therapy and diagnostic work up between Standard of Care (SOC) and Film Array Meningitis Encephalitis (FAME) groups

\begin{tabular}{|c|c|c|c|}
\hline Variables & $\operatorname{SOC}(n=52)$ & FAME $(n=46)$ & $P$ value \\
\hline Median in hospital length of stay (days) (IQR) & $18(7.5-28)$ & $17(12-27)$ & 0.786 \\
\hline Median ICU length of stay (days) (IQR) & $4(1-11)$ & $6(3-10)$ & 0.502 \\
\hline Glasgow Outcome Score ${ }^{a}$ & $3(1-4)$ & $4(1-4)$ & 0.214 \\
\hline In hospital mortality, $n$ (\%) & $17(32.7)$ & $15(32.6)$ & 0.993 \\
\hline Median time from test result to any therapy change (initiation or discontinuation) (hours, range) & $137.3(46-195)$ & $3(1.8-20.3)$ & $\leqslant 0.001$ \\
\hline Time from test result to initiation of appropriate therapy & $195.4(146.7-234.7)$ & $2.1(1.6-4.6)$ & $\leqslant 0.001$ \\
\hline Time from test result to discontinuation of inappropriate therapy & $92(47-190)$ & $19.1(2-71)$ & 0.050 \\
\hline Blood culture bottles $(>5)$ & $14(26.9)$ & $5(10.8)$ & 0.045 \\
\hline Head CT scan (>2) & $8(15.3)$ & $3(6.5)$ & 0.165 \\
\hline MRI of the brain & $11(21.1)$ & $19(41.3)$ & 0.031 \\
\hline
\end{tabular}

$\mathrm{IQR}$, interquartile range; ICU, intensive care unit; $\mathrm{CT}$, computed tomography; MRI, magnetic resonance image.

${ }^{a}$ Glasgow outcome score: a score of 1 indicates death, a score of 2 indicates a vegetative state (inability to interact with the environment), a score of 3 indicates severe disability (unable to live independently but follows commands), a score of 4 indicates moderate disability (unable to return to work or school but able to live independently), and a score of 5 indicates mild or no disability (able to return to work or school).

Table 5. Time (hours) to change in therapy between Standard of Care (SOC) and Film Array Meningitis-Encephalitis (FAME) groups according to microbiologic results

\begin{tabular}{|c|c|c|c|}
\hline $\begin{array}{l}\text { Time (hours) to change in therapy } \\
\text { (median, IQR) }\end{array}$ & SOC & FAME & $P$ value \\
\hline $\begin{array}{l}\text { If use of any antibiotic } \\
\text { (12 vs. 12) }\end{array}$ & $137(46.4-192)$ & $2.5(1.5-13.1)$ & $\leqslant 0.001$ \\
\hline If use of any antiviral ( 5 vs. 9) & $146.7(48.5-173)$ & $2(1.3-19.3)$ & 0.013 \\
\hline If use of Amphotericin B (5 vs. 6) & $195(92-217)$ & $2(1.2-6.1)$ & 0.010 \\
\hline $\begin{array}{l}\text { If neuroinfection discarded } \\
(1 \text { vs. } 4)\end{array}$ & 217 & $5(3-13.3)$ & 0.157 \\
\hline
\end{tabular}

IQR, interquartile range.

clinical suspicion of cryptococcosis is high not to discontinue antifungal therapy until a CSF cryptococcal latex antigen is obtained or the CSF culture is finalised [8]. The panel does not identify Epstein-Barr Virus (EBV), which has been related with neuronal inflammation, endothelial damage (even in the absence of clinical encephalitis) and central nervous system lymphoma in immunosuppressed patients, with impact in the development of cognitive dysfunction and psychiatric symptoms [14]. EBV is also associated with post-transplant lymphoproliferative disorder and occasionally with encephalitis in solid organ transplant patients' recipients [15].

In the SOC period, the aetiological diagnosis depended on the result of CSF culture, blood cultures as well as the PCR of viruses in CSF. The usual time to obtain cultures ranged between 3 and 5 days and that of the PCR between 14 and 21 days to be processed in reference laboratories outside of Pereira. During the 
Table 6. Antimicrobial use and duration between Standard of Care (SOC) and Film Array Meningitis-Encephalitis (FAME) groups

\begin{tabular}{|c|c|c|c|}
\hline $\begin{array}{l}\text { Antimicrobial therapy } \\
\text { use }(n, \%) \text { and duration } \\
\text { in days (median, IQR) }\end{array}$ & $\begin{array}{c}\text { SOC } \\
(n=52)\end{array}$ & $\begin{array}{c}\text { FAME } \\
(n=46)\end{array}$ & $\begin{array}{c}P \\
\text { value }\end{array}$ \\
\hline Use of any antibiotic & 32 (61.5) & $20(42.5)$ & 0.059 \\
\hline Duration of all antibiotics & $5(2-8.9)$ & $6.5(1-10.7)$ & 0.72 \\
\hline Ampicillin & $9(17.3)$ & $7(14.89)$ & 0.745 \\
\hline Duration & $1.5(0-6)$ & $4(0.5-8)$ & 0.296 \\
\hline Ceftriaxone & $23(44.2)$ & 15 & 0.239 \\
\hline Duration & $5(1-8)$ & (32.6) & 0.787 \\
\hline Cefepime & $8(15.4)$ & $6(0-9)$ & 0.067 \\
\hline Duration & $8(1-10)$ & $2(4.2)$ & 1.000 \\
\hline Meropenem & $8(15.4)$ & $10(0-20)$ & 0.067 \\
\hline Duration & $1(0-3.5)$ & $2(4.2)$ & 0.891 \\
\hline Vancomycin & $23(44.2)$ & $1(0-2)$ & 0.087 \\
\hline Duration & $6(1-10)$ & $13(27.6)$ & 0.767 \\
\hline Use of any antiviral & $14(26.9)$ & $5(1-8)$ & 0.332 \\
\hline Duration of all antiviral & $6.5(4.5-11)$ & $12(25.5)$ & 0.875 \\
\hline Acyclovir, & $13(25)$ & $11.5(3-15)$ & 0.401 \\
\hline Duration & $5.5(3-8)$ & $8(17.0)$ & 0.524 \\
\hline Gancyclovir & $1(1.9)$ & $5(3-12)$ & 0.135 \\
\hline Duration & $0(0-0)$ & $4(8.5)$ & 0.124 \\
\hline Amphotericin B & $16(30.77)$ & $16(0-20)$ & 0.184 \\
\hline Duration & $17(12-22)$ & $9(19.1)$ & 0.067 \\
\hline Antituberculous therapy & $9(17.3)$ & $\begin{array}{r}12.5(5-14) \\
14(29.8)\end{array}$ & 0.142 \\
\hline
\end{tabular}

$\mathrm{IQR}$, interquartile range.

FAME period, there was a significant improvement in the time to obtain the results that were associated with changes in empirical antimicrobial, both initiating appropriate therapy and discontinuing inappropriate therapy. The difference in costs of including the Biofire ${ }^{\varpi}$ FAME panel was not significant compared to SOC. There was a tendency of reduction in the cost of antimicrobial therapy and the cost of diagnostic studies with the implementation of the Biofire ${ }^{\circledast}$ FAME panel. To our knowledge, this is the first study documenting this impact of the FAME panel in Colombia. This also resulted in the practice of repeating blood cultures in the FAME period. A recent study evaluated the cost-effectiveness of setting up the ME panel, it was found that the main benefit from the economic point of view was in the decrease in the consumption of antimicrobials [16]. This finding contrasts with another study [10] where there was no timely suspension of inappropriate therapy according to the results of the panel, which reinforces the importance of the implementation of the test in the context of an antimicrobial stewardship programme.

Given the costs of the test, it is currently unclear if all CSF samples with suspected neuroinfection or only those with CSF pleocytosis should undergo testing with the panel. A paediatric study showed that EV may present without pleocytosis in infants suggesting that it may be cost-effective in a universal approach with the ME panel by reducing the number of studies and days of hospitalisation [17].

Limitations of the study included the limited number of patients who underwent the test, the completion of the study in a single centre and the non-confirmation of the positive results of the virus with another test. Furthermore, the sample size also did not allow a differential analysis of results by type of aetiology, which may show differences with respect to the global analysis and possibly was associated with a low power in detecting an impact on clinical outcomes.

As for strengths, this study is the first to evaluate the clinical impact of the implementation of the Biofire ${ }^{\oplus}$ FAME panel test in Colombia as part of the study plan of adult patients with clinical suspicion of neuroinfection. Given the results presented, it is considered that the test is useful in the study of patients with neuroinfection mainly in the reduction of antimicrobial consumption in the context of an antibiotic control programme and directed according to alterations of the CSF cytochemistry.

In conclusion, the implementation of the FAME panel in a public hospital in Colombia resulted in a more rapid diagnosis that improved the detection of pathogens and had an impact on appropriately modifying the empirical antimicrobial management of patients but had no impact of lengths of stay or outcomes. Future studies should validate these results in other Latin American countries.

Financial support. Grant A Star Foundation.

Conflict of interest. RH has research support and is a speaker for Biofire ${ }^{\circ}$. All other authors have no conflicts of interest.

Ethical standards. The study was approved by the Institutional Review Board of the Hospital San Jorge de Pereira.

Data availability statement. The data for the study are available by contacting the corresponding author.

\section{References}

1. Edmond K et al. (2010) Global and regional risk of disabling sequelae from bacterial meningitis: a systematic review and meta-analysis. Lancet Infectious Diseases 10, 317-328.

2. Tunkel AR et al. (2008) The management of encephalitis: clinical practice guidelines by the Infectious Diseases Society of America. Clinical Infectious Diseases 47, 303-327.

3. Tunkel AR et al. (2004) Practice guidelines for the management of bacterial meningitis. Clinical Infectious Diseases 39, 1267-1284.

4. Fitch MT et al. (2007) Emergency diagnosis and treatment of adult meningitis. Lancet Infectious Diseases 7, 191-200.

5. Brouwer MC et al. (2010) Epidemiology, diagnosis, and antimicrobial treatment of acute bacterial meningitis. Clinical Microbiology Review 23, 467-492.

6. Dubberke ER et al. (2008) Short- and long-term attributable costs of Clostridium difficile-associated disease in nonsurgical inpatients. Clinical Infectious Diseases 46, 497-504.

7. Leber A et al. (2015) Multi-center clinical evaluation of a multiplex meningitis/encephalitis PCR panel for simultaneous detection of bacteria, yeast, and viruses in cerebrospinal fluid specimens. Journal Clinical Microbiology 54, 2251-2261.

8. Liesman RM et al. (2018) Evaluation of a commercial multiplex molecular panel for diagnosis of infectious meningitis and encephalitis. Journal Clinical Microbiology 56, 1-9.

9. Naccachea SN et al. (2018) One year in the life of a rapid syndromic panel for meningitis/encephalitis: a pediatric tertiary care facility's experience. Journal Clinical Microbiology 56, e01940-17. 
10. Chang M et al. (2018) Tertiary care center's experience with novel molecular meningitis/encephalitis diagnostics and implementation with antimicrobial stewardship. Military Medicine 183, 8-11.

11. Tarai B et al. (2018) Filmarray ${ }^{\circledR}$ meningitis/encephalitis (ME) panel, a rapid molecular platform for diagnosis of CNS infections in a tertiary care hospital in North India: one-and-half-year review. Neurology Sciences 40, 81-88.

12. Piccirilli G et al. (2018) Infectious meningitis/encephalitis: evaluation of a rapid and fully automated multiplex PCR in the microbiological diagnostic workup. New Microbiology 41, 118-125.

13. Rhein J et al. (2016) Diagnostic performance of a multiplex PCR assay for meningitis in an HIV infected population in Uganda. Diagnostic Microbiology Infectious Diseases 84, 266-273.
14. Lupia T et al. (2020) Presence of Epstein-Barr virus DNA in cerebrospinal fluid is associated with greater HIV RNA and inflammation. AIDS (London, England) 34, 373-380.

15. Lau JSY et al. (2017) Epstein-Barr virus encephalitis in solid organ transplantation. New Microbiology 40, 212-217.

16. Soucek D et al. (2019) Cost justification of the BioFire FilmArray meningitis/encephalitis panel versus standard of care for diagnosing meningitis in a community hospital. Journal Pharmacy Practice 32, 36-40.

17. Lumley SF et al. (2018) Multiplex PCR reveals high prevalence of enterovirus and HHV6 in acellular paediatric cerebrospinal fluid samples. Journal of Infection 77, 251-225. 